\title{
Nanopatterned Graphene on a Polymer Substrate by a Direct Peel- off Technique
}

\author{
T. L. Chen, ${ }^{* \dagger}$ D. S. Ghosh, ${ }^{* \dagger}$ M. Marchena, ${ }^{\dagger}$ J. Osmond, $^{\dagger}$ and V. Pruneri ${ }^{\dagger, \ddagger}$ \\ ${ }^{\dagger}$ ICFO-Institut de Ciències Fotòniques, Mediterranean Technology Park, 08860 Castelldefels, Barcelona, Spain \\ ${ }^{\ddagger}$ ICREA-Institució Catalana de Recerca i Estudis Avançats, 08010 Barcelona, Spain
}

Supporting Information

ABSTRACT: A graphene ( $\mathrm{Gr}$ ) on a polyimide (PI) polymer film (Gr-PI film), obtained by a direct peel-off technique, is proposed and investigated. Thanks to its high transparency, electrical conductivity, mechanical strength, and chemical durability, the Gr-PI film is an ideal substrate for flexible electronic and optoelectronic devices, including transistors, lightemitting diodes, and plasmonic antennas. It is obtained using a straightforward method. After spin coating and curing a PI film on $\mathrm{Gr}$ previously grown on $\mathrm{Cu}$, one can separate the $\mathrm{Gr}-\mathrm{PI}$ film from the $\mathrm{Cu}$ foil thanks to the difference in the adhesive energy
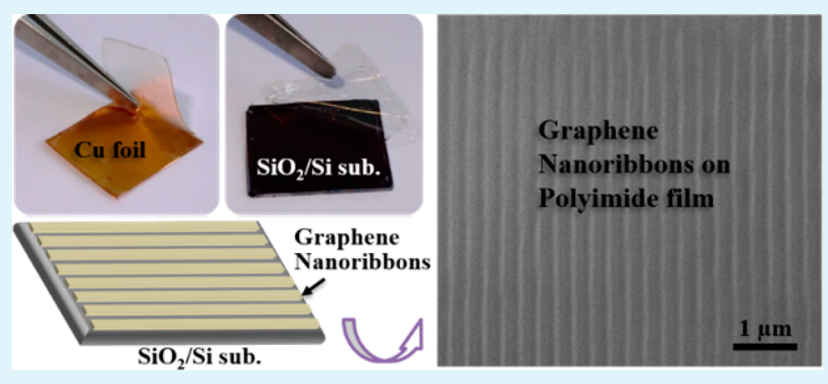
between the $\mathrm{Gr}-\mathrm{Cu}$ and $\mathrm{Gr}-\mathrm{PI}$ interfaces. The resulting $\mathrm{Gr}-\mathrm{PI}$ film shows an average electrical sheet resistance ranging from 520 to $860 \Omega /$ sq and a very high optical transmission (>90\%), which have allowed the demonstration of a transparent heater. The surface morphology of the Gr-PI film follows that of the $\mathrm{Cu}$ foil, with the latter maintaining its surface properties and allowing in this way its reuse in subsequent chemical vapor deposition growth. The method can also be applied to patterned $\mathrm{Gr}$, as is demonstrated for nanosize ribbons with a width of a few tens of nanometers.

KEYWORDS: peel-off, graphene transfer, graphene pattern, polymer substrate, flexible substrate

\section{INTRODUCTION}

In recent years, graphene $(\mathrm{Gr})$ has attracted enormous interest because of its unique physical and chemical properties. ${ }^{1-3}$ It is worth noting that chemical vapor deposition (CVD) has paved the way to industrial production of $\mathrm{Gr}^{4-6}$ To date, the majority of $\mathrm{Gr}$ research and development has focused on rigid substrates such as $\mathrm{SiO}_{2}$ on $\mathrm{Si}, \mathrm{SiC}$, or glass. ${ }^{7-10}$ However, there is a strong need in using flexible substrates, such as poly(ethylene terephthalate) and thin glass, for making devices that can be bent or even folded and being able to use inexpensive roll-toroll fabrication techniques. This is the case, for example, for organic solar cells (OSCs), organic light-emitting diodes, tactile displays, and plasmonic nanoantennas. ${ }^{11} \mathrm{Gr}$ needs then to be directly grown or transferred onto the flexible substrates. Transfer is still preferred for the high-quality layers obtained on catalytic metals, e.g., $\mathrm{Cu}, \mathrm{Pt}$, and $\mathrm{Ni}$, using CVD. Poly(methyl methacrylate) (PMMA) is used as a supporting layer in wetsolution transfer but an adhesive layer in hot-pressing/ lamination. ${ }^{12-15}$ Besides an additional intermediate layer, an etching process is usually needed to remove the underlying $\mathrm{Cu}$ foil, which would thus be wasted. A poly(dimethylsiloxane) (PDMS) polymer was used as a sacrificial solid block to facilitate the handling process during a wet-etching process. ${ }^{16-18}$ In another experiment, it was also used to exfoliate Gr from another Gr layer thanks to the weak adhesive force between neighboring Gr layers. ${ }^{19}$ However, PDMS has never been used for peeling off $\mathrm{Gr}$ grown on $\mathrm{Cu}$ because the bonding force that one has to overcome in this case is much higher than that between Gr layers. Recently, in the case of the hotpressing/lamination method, water has been proposed to avoid etching because it can penetrate through a capillary effect between $\mathrm{Gr}$ and the adhesive layer to partially separate them. The price of $\mathrm{Cu}$ foil is quite high, a practical limit for the industrial use of CVD-grown $\mathrm{Gr}$ if it is not reused.

In this paper, a new Gr on a polyimide (PI) film (Gr-PI film) is proposed and investigated as a substrate for electronic and optoelectronic applications. PI films have already been employed as durable and flexible substrates or intermediate layers in microelectronics, OSCs, and aerospace applications thanks to their high transparency, chemical and mechanical resistance, and easy processing. ${ }^{20}$ Several scalable fabrication techniques exist for depositing PI over large areas such as Doctor blade, Meyer rod, or spray-coating methods. ${ }^{21-24}$ The $\mathrm{Gr}-\mathrm{PI}$ film is obtained by using an etching-free transfer method consisting of depositing a layer of PI on Gr previously grown on a $\mathrm{Cu}$ substrate and peeling the $\mathrm{Gr}$ off thanks to the fact that the interface binding energy between PI and Gr is larger than that between the $\mathrm{Cu}$ foil and Gr. The resulting $\mathrm{Gr}-$ PI film has high optical transparency and electrical conductivity. In addition, the integrity of the $\mathrm{Cu}$ foil is preserved after the

Received: January 7, 2015

Accepted: February 24, 2015

Published: February 24, 2015 
peel-off. Subsequently, the $\mathrm{Cu}$ foil was flattened and reused to deposit a new monolayer $\mathrm{Gr}$ on its top by following the same CVD process.

\section{EXPERIMENTAL DETAILS}

Synthesis of a Gr Film. Gr films were grown on 35- $\mu$ m-thick $\mathrm{Cu}$ foils (BHZ-Z-HA, JX Nippon Mining \& Metals) cut into $1 \times 1$ in. $^{2}$ dimensions. The $\mathrm{Cu}$ foil was first cleaned with acetone, isopropyl alcohol, and acetic acid for 20, 20, and 3 min, respectively. Then, the $\mathrm{Cu}$ foil was placed into the PE-CVD chamber (Black Magic 4 in., AIXTRON). When the vacuum reached $0.1 \mathrm{mbar}, \mathrm{N}_{2}$ and Ar flows were introduced into the system at $250: 250 \mathrm{sccm}$ ratios, respectively. Meanwhile, a pretreatment with $\mathrm{H}_{2}(1000 \mathrm{sccm})$ is implemented for the $\mathrm{Cu}$ foil from room temperature to the reaction temperature, which is very beneficial for removing any residual oxide and increasing the grain size of $\mathrm{Cu}$. After a slow ramping up to $1000{ }^{\circ} \mathrm{C}$, the reaction gases were fed into the system $\left(\mathrm{CH}_{4}, 15 \mathrm{sccm} ; \mathrm{H}_{2}, 60 \mathrm{sccm}\right)$ while keeping the pressure at a constant value of $25 \mathrm{mbar}$. For full coverage of a $1 \times 1$ in. $^{2}$ substrate, the optimum reaction time was $7.5 \mathrm{~min}$. Finally, the sample was cooled with a low $\mathrm{H}_{2}$ pressure in order to remove any unwanted amorphous $\mathrm{C}$ from the surface.

Fabrication of Gr-PI. Initially, a PI polymer solution (VTEC-080051) was spin-coated onto $\mathrm{Gr}$ on $\mathrm{Cu}$ foil ( $\mathrm{Gr} / \mathrm{Cu}$ foil) and subsequently annealed at $100{ }^{\circ} \mathrm{C}$ for $10 \mathrm{~min}$, followed by another step at $180^{\circ} \mathrm{C}$ for $10 \mathrm{~min}$. The annealing process can dry and solidify PI into a thin film. PI was then peeled-off from the $\mathrm{Cu}$ foil to obtain a solid, smooth, and free-standing flexible Gr-PI film with Gr firmly attached to PI. The thickness of the PI film can be controlled by adjusting the spin-coater speed. In this work, by using a coating speed of $1000 \mathrm{rpm}$, the thickness of the PI film was about $5 \mu \mathrm{m}$.

Fabrication of Gr Nanoribbons. Gr nanoribbons were fabricated on a $\mathrm{SiO}_{2} / \mathrm{Si}$ substrate by electron-beam lithography and reactive ion etching. An electron-beam negative resist (Allresist AR-N 7500.08) was spin-coated at $4000 \mathrm{rpm}$ for a thickness of around $100 \mathrm{~nm}$ on a $\mathrm{Gr} / \mathrm{SiO}_{2} / \mathrm{Si}$ substrate and successively baked at $90{ }^{\circ} \mathrm{C}$ for $1 \mathrm{~min}$. The AR-N resist was exposed to a $50 \mathrm{kV}$ electron beam at $25 \mathrm{pA}$ current (Crestec CABL 9000C). Gr was then patterned with $\mathrm{Ar} / \mathrm{O}_{2}$ reactive ion etching. The AR-N resist was successively removed with NEP solvent soaking.

Characterization. A PerkinElmer Lambda 950 spectrometer was used for optical transmission measurements, while a four-point probe and a standard Van der Pauw method was used for electrical sheet resistance measurements. The surface morphology of the films was characterized by optical and atomic force microscopy (AFM; D3100). The quality of Gr was assessed using Raman spectroscopy (Renishaw InVia Raman microscope).

Transparent Heater. A source with a potentiometer was used to apply the voltage. The temperature was recorded as a function of time using a Fluke 566 IR thermometer. The temperature distribution images of the heater were taken by an IR camera.

\section{RESULTS AND DISCUSSION}

Figure 1 shows the schematic steps of the proposed etchingfree $\mathrm{Gr}$ transfer process, where the $\mathrm{Cu}$ foil can be reused to deposit a new monolayer $\mathrm{Gr}$ on its top by following the same CVD process.

Figure 2a shows a Raman mapping image of as-grown CVD of $\mathrm{Gr}$ on $\mathrm{Cu}$ foil. The average peak intensity ratio between the $2 \mathrm{D}$ and $\mathrm{G}$ bands is about 2.8 without any appearance of a defect-related $\mathrm{D}$ band over the whole scanning area $(10 \times 10$ $\mu \mathrm{m}^{2}$ ), indicating a high-quality monolayer structure. Figure $2 \mathrm{~b}$ shows an optical microscopic image of PI on $\mathrm{Gr} / \mathrm{Cu}$ foil. The same figure (right-hand side) shows that the PI thin film could be easily peeled off from $\mathrm{Cu}$ foil. This simple peel-off process allows one to transfer $\mathrm{Gr}$ without any cumbersome $\mathrm{Cu}$ etching step. As was mentioned previously, the underlying $\mathrm{Cu}$ foil can be flattened and reused. Figure $2 c$ shows two Raman spectra

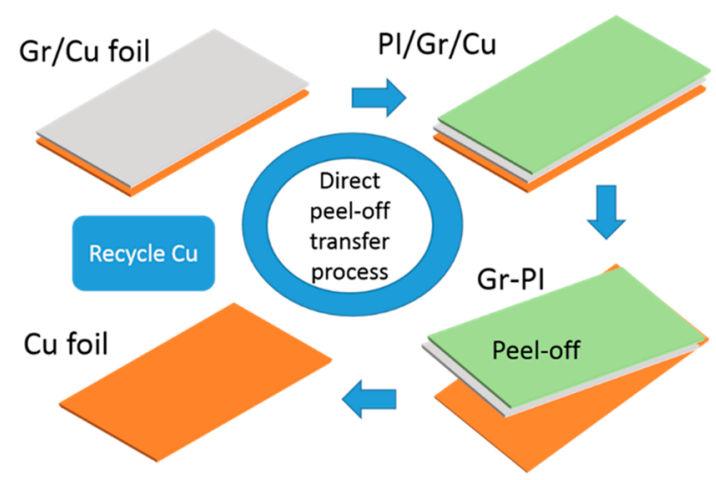

Figure 1. Steps of the proposed etching-free $\mathrm{Gr}$ transfer process based on peel-off after deposition of $\mathrm{Gr}$ on a $\mathrm{Cu}$ substrate and solidification of a PI film.

(point scans) for it before and after the peel-off process. Typical bands for $\mathrm{Gr}$ (2D and $\mathrm{G}$ peaks) are clearly present before the peel-off process, two defect-related peaks ( $\mathrm{D}$ and $\mathrm{D}^{\prime}$ bands) are almost negligible, and also the $2 \mathrm{D} / \mathrm{G}$ ratio gives a value of about 3.1, indicating a high-quality monolayer structure. In contrast, after the peel-off process, there is no sign of any Raman peak, essentially resembling the typical spectrum of bare $\mathrm{Cu}$ foil.

These trends were also confirmed over the full scanned area of about $50 \times 50 \mu \mathrm{m}^{2}$. The damage (ablation) threshold of the ultrathin PI film under laser irradiation is quite low (see S1 in the Supporting Information, SI); therefore, direct detection of Gr on the PI film by a Raman technique was only performed in a low-laser-intensity regime and for a short exposure time. Figure 3 shows the Raman spectra for PI and Gr-PI, respectively. It can be clearly seen that the $2 \mathrm{D}$ peak is present in the $\mathrm{Gr}-\mathrm{PI}$ sample. However, because of a large background noise from the PI film, the typical G band could not be detected because it overlaps with significant noise (see also the spectrum for only the PI film, which confirms the large noise from the substrate). To investigate further the uniformity of the Gr layer on PI, we extended the Raman mapping scan over an area of about $10 \times 10 \mu \mathrm{m}^{2}$ to examine the $2 \mathrm{D}$ peak intensity distribution. As is shown in the inset of Figure 3, a relatively high intensity of the $2 \mathrm{D}$ peak is present over the whole detected area, a strong indication of a continuous Gr layer. Similar mapping scans were performed over several randomly selected areas and gave consistent results. Therefore, one can conclude that the $\mathrm{Gr}$ grown on $\mathrm{Cu}$ foil is entirely transferred to the PI film.

The Gr-PI film shows an important feature: that is, its surface morphology reproduced the initial $\mathrm{Cu}$ foil morphology (Figure $4 \mathrm{a}, \mathrm{b}$ ). This is another confirmation that the $\mathrm{Gr}-\mathrm{PI}$ layer was peeled off completely without any damage. CVDgrown $\mathrm{Gr}$ on a thicker $\mathrm{Cu}$ plate was also tested, and the Raman data given in S2 in the SI show the typical Gr bands despite a large $\mathrm{Cu}$ plate thickness $(2 \mathrm{~mm})$. Subsequently, the peel-off process was applied to this case, and the result is quite similar to the case of $\mathrm{Cu}$ foil.

The same peel-off process was also successfully applied to $\mathrm{Gr}$ transferred on a glass substrate via the PMMA-assisted method (see S3 in the SI). Several groups have reported that the adhesive energies in $\mathrm{Gr}$-glass and $\mathrm{Gr}-\mathrm{Cu}$ are about 0.45 and $0.72 \mathrm{~J} \mathrm{~m}^{-2}$, respectively. ${ }^{25-27}$ Although the bonding type has a van der Waals nature for both cases, the higher force for the latter substrate is believed to be associated with a much higher 

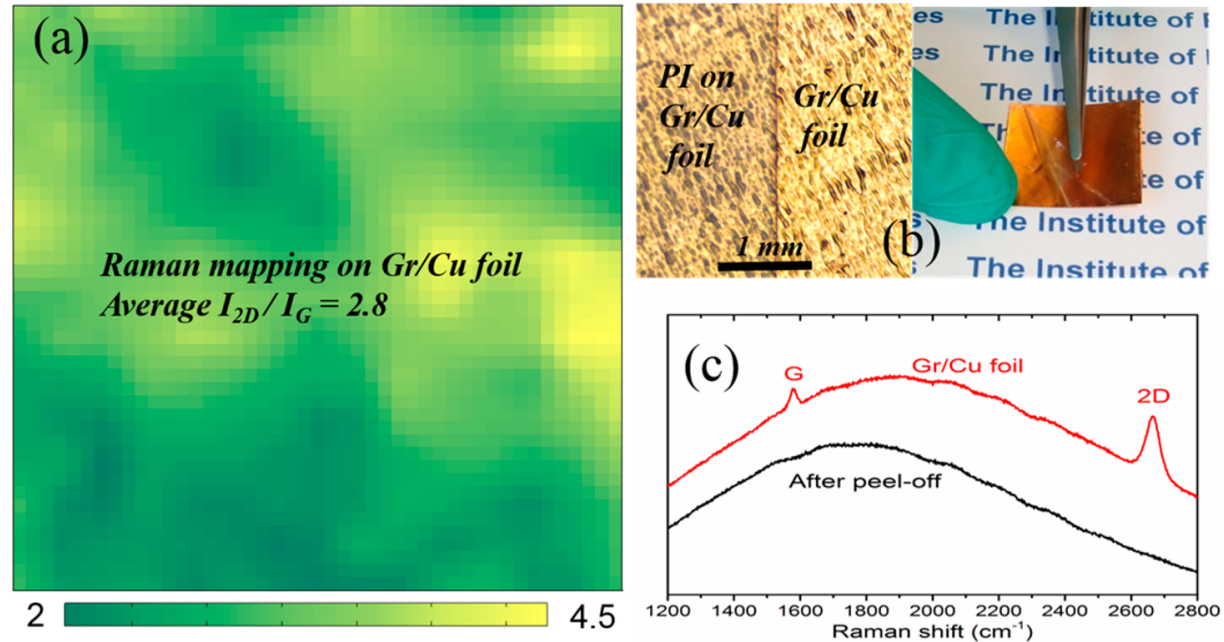

Figure 2. Raman mapping image of as-grown CVD of Gr on Cu foil with a scanning area of $10 \times 10 \mu \mathrm{m}^{2}$ (a). Optical microscopic image of PI on $\mathrm{Gr} / \mathrm{Cu}$. The same figure (right panel) demonstrates that the PI thin film can be easily peeled off from Cu foil (b). Two Raman spectra (point scans) before and after the peel-off process (c).

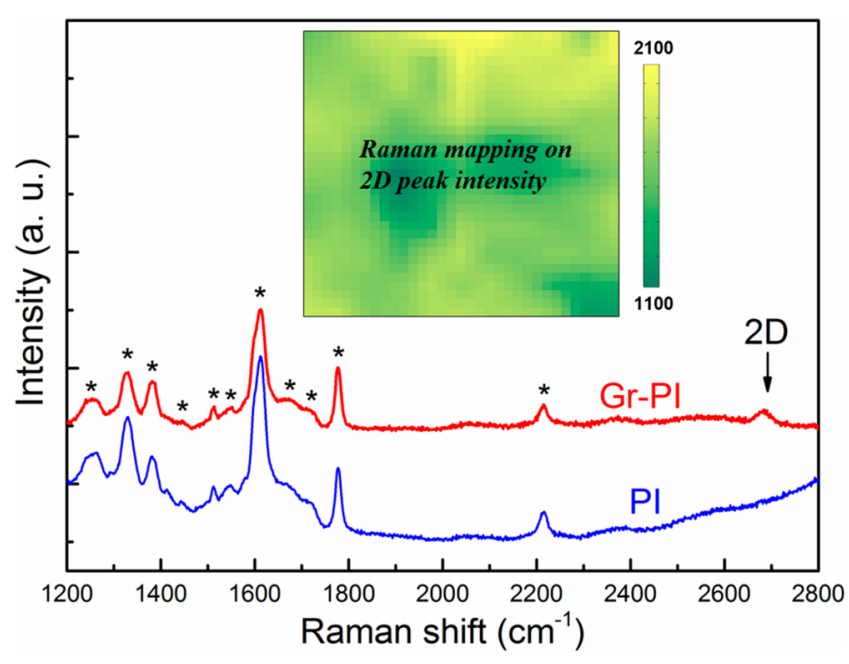

Figure 3. Raman spectra for PI and Gr-PI, respectively. Asterisks represent the Raman signals from a background PI film. The inset is a Raman mapping image for the Gr $2 \mathrm{D}$ peak intensity over a scanning area of about $10 \times 10 \mu \mathrm{m}^{2}$.
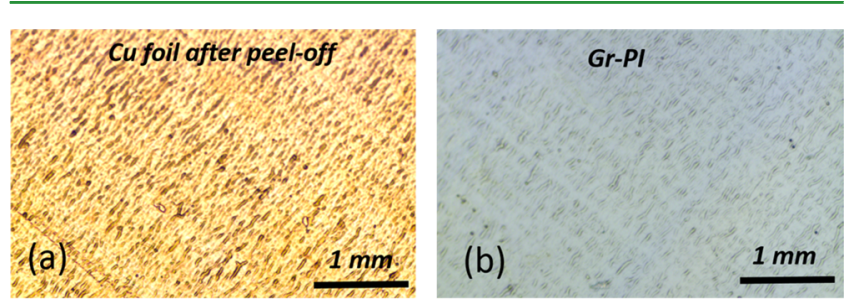

Figure 4. Demonstration that the peeled-off Gr-PI film can reproduce the surface morphology of the initial $\mathrm{Cu}$ foil: morphology of $\mathrm{Cu}$ foil after the peel-off process (a); morphology of the Gr-PI film (b).

electronic density at the interface. Regardless, our experiments suggest that the adhesion force of $\mathrm{Gr}-\mathrm{PI}$ is higher than that between the "Gr-Cu foil" and "Gr-glass" interfaces. The transfer can be attributed to a uniform wetting process of a liquid-solid (PI on Gr) interface, which is then solidified during the PI thermal curing, resulting in a stronger adhesion force than that of the solid-solid ( $\mathrm{Gr}$ on $\mathrm{Cu}$ or $\mathrm{Gr}$ on glass) interface generated during CVD or transfer.
As for the case of $\mathrm{Gr}$ on $\mathrm{Cu}$, for $\mathrm{Gr}$ on glass the peel-off process replicated the surface morphology of the glass substrate, i.e., its smoothness, in the resulting Gr-PI film. A comparison of the AFM images of the Gr-PI films peeled off from $\mathrm{Cu}$ foil and glass substrate indicates that the $\mathrm{Gr}-\mathrm{PI}$ film peeled off from $\mathrm{Cu}$ foil has a much rougher surface morphology (see S3 in the SI). The surface roughness produces light scattering, with this feature being unwanted in several applications, for example, displays where high visibility (clarity) is required, but welcomed in others, for example, thin film solar cells, where it can be used to enhance light trapping and harvesting. ${ }^{28-30}$ Figure 5 shows the direct optical transmittance

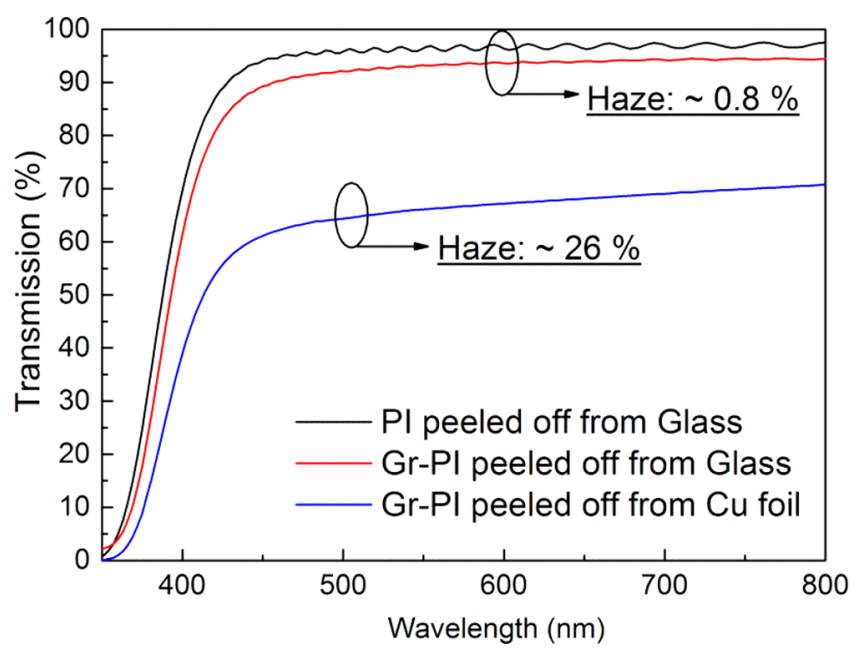

Figure 5. Direct optical transmittance and average haze of the samples: PI film peeled off from glass substrate, Gr-PI film peeled off from glass substrate, and $\mathrm{Gr}-\mathrm{PI}$ film peeled off from $\mathrm{Cu}$ foil.

and average haze of the samples: the PI film peeled off from glass substrate, the Gr-PI film peeled off from glass substrate, and the $\mathrm{Gr}-\mathrm{PI}$ film peeled off from $\mathrm{Cu}$ foil. The PI film itself was highly transparent $(>95 \%)$ with little haze $(<1 \%)$. The GrPI film peeled-off from glass substrate maintained the low haze, while its transmission decreased about $2-3 \%$ because of the absorption of Gr. The $\mathrm{Gr}-\mathrm{PI}$ film peeled off from $\mathrm{Cu}$ foil had a 

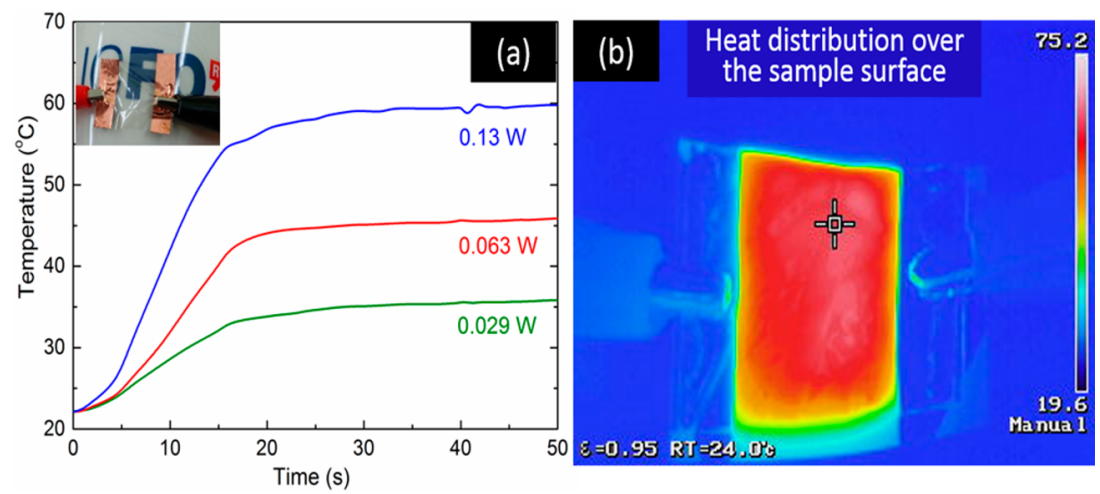

Figure 6. (a) Temperature profiles as a function of time for the Gr-PI film for different electrical power (the inset shows a real picture of the sample used in the measurement). (b) Typical IR image for temperature distribution taken by an IR camera.

much lower direct transmission (about 65-70\%) because the rest of the light is scattered off the collecting angle of the detector. This is confirmed by the significantly larger haze (26\%).

The electrical sheet resistance of the peeled-off Gr-PI film was evaluated by a standard Van der Pauw method and gave values ranging from 520 to $860 \Omega /$ sq. To demonstrate the effective electrical conduction as well as uniformity of the transferred Gr layer on the PI film, the Gr-PI film was used to fabricate a transparent thin-film heater (TTFH) based on Joule's effect. TTFHs are very versatile for smart windows and displays, acting as antifogging and deicing surfaces. Because of the outstanding thermal conductivity of Gr, Gr-based TTFHs can give an extra advantage. In this work, the active area of the Gr-PI-based TTFH was $1 \times 1$ in. $^{2}$ (inset of Figure 6a). The temperature was recorded for different electrical powers over about $60 \mathrm{~s}$. The temperature increased quite rapidly toward a saturation level (Figure 6a). The temperature uniformity of the Gr-PI-based TTFH sample was confirmed by taking an IR image when the maximum temperature was reached (Figure $6 \mathrm{~b})$, which further confirms the uniformity and continuity of the transferred Gr. In addition, the mechanical robustness and surface conduction of the Gr-PI film were found to be high by continuous bending and twisting in any direction without observation of any degradation in the conduction properties (video M1 in the SI).

To demonstrate that $\mathrm{Cu}$ foil could be reused after peeling off from the Gr-PI film, the same foil was flattened by a plain cold press (pressure of $100 \mathrm{psi}$ ) and cleaned with acetone, isopropyl alcohol, and acetic acid for 20, 20, and $3 \mathrm{~min}$, respectively. Subsequently, $\mathrm{Cu}$ foil was placed in the plasma-enhanced CVD chamber for Gr growth using the same conditions as those of the first growth. Figure 7a shows the Raman spectrum for the new $\mathrm{Gr}$ layer on the reused $\mathrm{Cu}$ foil, providing evidence of its high quality.

The new Gr layer was transferred to a standard $\mathrm{SiO}_{2} / \mathrm{Si}$ substrate by a conventional PMMA-assisted method. ${ }^{31}$ In this case, the underlying $\mathrm{Cu}$ foil was etched away by floating the $\mathrm{PMMA} / \mathrm{Gr} / \mathrm{Cu}$ sandwich structure in a concentrated $\mathrm{FeCl}_{3}$ solution $(1 \mathrm{M})$ at room temperature for about $3.5 \mathrm{~h}$, and then the $\mathrm{Gr}-\mathrm{PMMA}$ film was rinsed in deionized water and directly transferred onto a $\mathrm{SiO}_{2} / \mathrm{Si}$ substrate. Finally, PMMA was completely dissolved with acetone. Figure 7 a shows the Raman spectrum for the transferred $\mathrm{Gr}$ on a $\mathrm{SiO}_{2} / \mathrm{Si}$ substrate, exhibiting a monolayer $\mathrm{Gr}$ with excellent quality $\left(I_{2 \mathrm{D}} / I_{\mathrm{G}}=2.3\right.$ and $\left.I_{\mathrm{D}} / I_{\mathrm{G}}=0.11\right)$. Because of a clear optical contrast, the

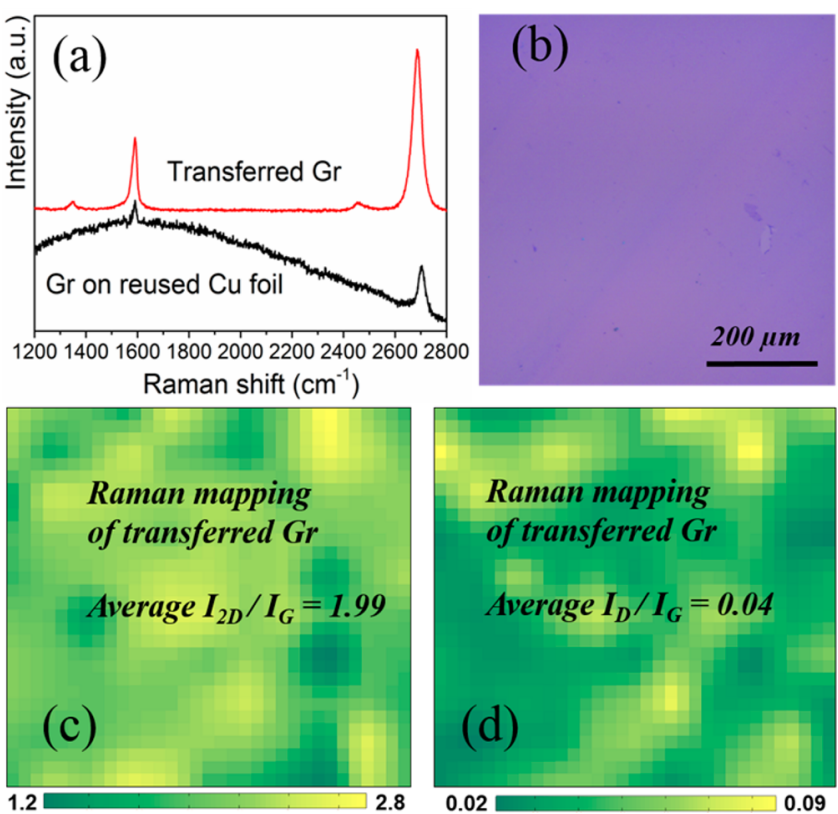

Figure 7. (a) Raman spectra for the new Gr layer on the reused $\mathrm{Cu}$ foil and for the transferred $\mathrm{Gr}$ on a $\mathrm{SiO}_{2} / \mathrm{Si}$ substrate. (b) Optical microscopy image of the transferred Gr. (c and d) Raman mapping images for peak intensity ratios of $2 \mathrm{D} / \mathrm{G}$ and $\mathrm{D} / \mathrm{G}$, respectively. The scanning area is $10 \times 10 \mu \mathrm{m}^{2}$.

transferred $\mathrm{Gr}$ on $\mathrm{SiO}_{2} / \mathrm{Si}$ could be visualized effectively through an optical microscope (Figure $7 \mathrm{~b}$ ), showing an almost crack/wrinkle-free and clean Gr surface. The Raman mapping scan over the $10 \times 10 \mu \mathrm{m}^{2}$ area also provided more evidence about the quality of the transferred Gr. Parts $\mathrm{c}$ and d of Figure 7 indicate an average peak intensity ratio between the $2 \mathrm{D}$ and $\mathrm{G}$ bands of about 2, while the one between the $D$ and $G$ bands is almost negligible (only 0.04). If one plots the distribution of $I_{2 \mathrm{D}} / I_{\mathrm{G}}$ over the scanning area into a histogram, one can clearly observe that the centered peak value is about 3 (see S4 in the SI). All of these measurements confirm the high quality of the Gr layer and that the proposed peel-off process allows one to reuse $\mathrm{Cu}$ foil as the substrate for subsequent growth.

In order to assess the potential of the proposed Gr-PI film and its fabrication method for light and flexible electronics and optoelectronics, micro- and nanopatterns of $\mathrm{Gr}$ were also demonstrated because they are often required to realize devices. One should note that common patterning techniques face challenges during processing of a flexible polymer substrate 
because of its deformation and instability. ${ }^{32-34}$ In this paper, we first patterned a $\mathrm{Gr}$ nanoribbon structure onto a $\mathrm{SiO}_{2} / \mathrm{Si}$ substrate by electron-beam lithography. By using the peel-off process previously described, the Gr nanoribbons were directly transferred onto the PI film. Figure 8a shows the schematic of
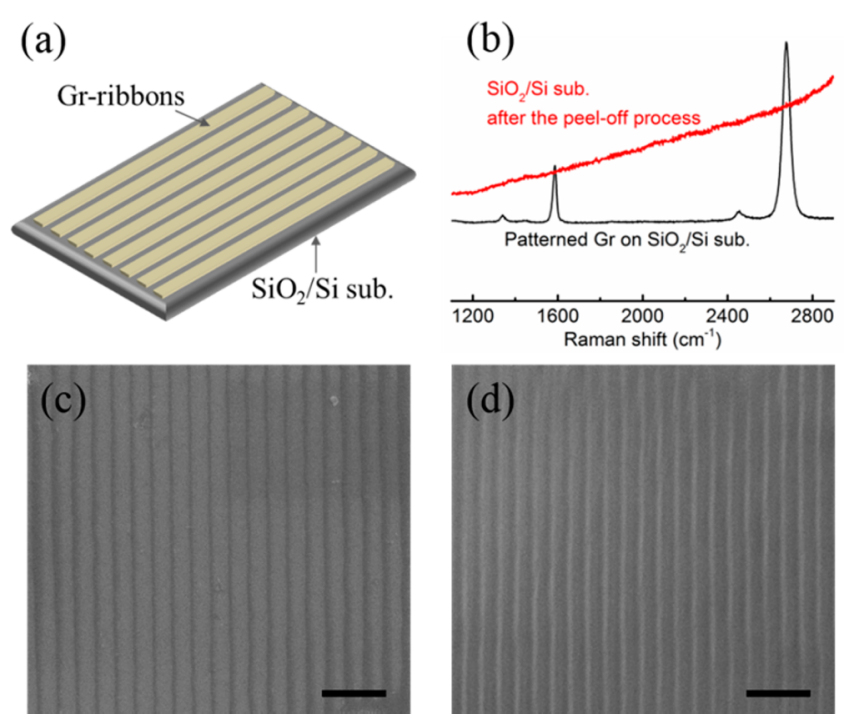

Figure 8. (a) $\mathrm{Gr}$ nanoribbons on a $\mathrm{SiO}_{2} / \mathrm{Si}$ substrate, (b) Raman signals from the same area of the $\mathrm{SiO}_{2} / \mathrm{Si}$ substrate before and after the peel-off process. (c and d) SEM images of Gr nanoribbons before and after the peel-off process for the $\mathrm{SiO}_{2} / \mathrm{Si}$ and $\mathrm{PI}$, respectively. The scale bar is $1 \mu \mathrm{m}$.

Gr nanoribbons on a $\mathrm{SiO}_{2} / \mathrm{Si}$ substrate. A typical Gr Raman signal was detected before the process, while it was absent within the same area on the $\mathrm{SiO}_{2} / \mathrm{Si}$ substrate after the peel-off process (as shown in Figure $8 \mathrm{~b}$ ). Parts $\mathrm{c}$ and $\mathrm{d}$ of Figure 8 are the SEM images before and after the peel-off process for the $\mathrm{SiO}_{2} / \mathrm{Si}$ and PI substrates, respectively. This clearly demonstrates the versatility of the proposed method to achieve patterned $\mathrm{Gr}$ on polymer, even at nanoscale dimensions.

\section{CONCLUSIONS}

A new Gr on the PI film has been proposed as a flexible polymer substrate with high transparency, electrical conductivity, and mechanical and chemical stability for electronic and optoelectronic applications. A transparent heater based on the developed film was demonstrated. The technique to produce it consisted of a simple peel-off process after deposition and solidification of PI on top of $\mathrm{Gr}$ on a $\mathrm{Cu}$ substrate. Cumbersome and not always clean etching required in common transfer processes could be avoided. In addition, $\mathrm{Cu}$ foil maintained its surface morphology and chemistry after the process, making its reusage possible and lowering in this way the costs. We also showed that patterned Gr down to the nanoscale could be achieved on a PI film by using the same process. This can be important for nanoelectronic and nanooptic devices including miniature, fast transistors and optical antennas.

\section{ASSOCIATED CONTENT}

\section{S Supporting Information}

Ablation effect during the direct detection of Gr on the PI film by a Raman technique (S1), Raman spectrum for Gr grown on a thicker $\mathrm{Cu}$ plate by CVD (S2), peel-off process from $\mathrm{Gr}$ transferred onto a glass substrate (S3), a histogram distribution of the Gr peak intensity ratio of $2 \mathrm{D} / \mathrm{G}$ over the scanning area for the transferred $\mathrm{Gr}$ (S4), and a demonstration of the mechanical robustness and surface conduction of the $\mathrm{Gr}-\mathrm{PI}$ film (video M1). This material is available free of charge via the Internet at http://pubs.acs.org.

\section{AUTHOR INFORMATION}

\section{Corresponding Authors}

*E-mail: tonglai.chen@icfo.es.

*E-mail: dhriti.ghosh@icfo.es.

\section{Notes}

The authors declare no competing financial interest.

\section{ACKNOWLEDGMENTS}

We acknowledge financial support from the Spanish Ministry of Economy and Competitiveness and "Fondo Europeo de Desarrollo Regional” through Grant TEC2013-46168-R. Partial support was also received by Corning Inc. T.L.C. acknowledges financial support through a Ramon y Cajal fellowship.

\section{REFERENCES}

(1) Geim, A. K.; Novoselov, K. S. The Rise of Graphene. Nat. Mater. 2007, 6, 183-191.

(2) Geim, A. K. Graphene: Status and Prospects. Science 2009, 324, 1530-1534.

(3) Neto, A. C.; Guinea, F.; Peres, N. M. R.; Novoselov, K. S.; Geim, A. K. The Electronic Properties of Graphene. Rev. Mod. Phys. 2009, 81, 109.

(4) Zhang, Y.; Zhang, L.; Zhou, C. Review of Chemical Vapor Deposition of Graphene and Related Applications. Acc. Chem. Res. 2013, 46, 2329-2339.

(5) Vlassiouk, I.; Fulvio, P.; Meyer, H.; Lavrik, N.; Dai, S.; Datskos, P.; Smirnov, S. Large Scale Atmospheric Pressure Chemical Vapor Deposition of Graphene. Carbon 2013, 54, 58-67.

(6) Chen, T. L.; Ghosh, D. S.; Mkhitaryan, V.; Pruneri, V. Hybrid Transparent Conductive Film on Flexible Glass Formed by HotPressing Graphene on a Silver Nanowire Mesh. ACS Appl. Mater. Interfaces 2013, 5, 11756-11761.

(7) Bonaccorso, F.; Sun, Z.; Hasan, T.; Ferrari, A. C. Graphene Photonics and Optoelectronics. Nat. Photonics 2010, 4, 611-622.

(8) Zhou, S. Y.; Gweon, G. H.; Fedorov, A. V.; First, P. N.; De Heer, W. A.; Lee, D. H.; Guinea, F.; Castro, N. A. H.; Lanzara, A. SubstrateInduced Bandgap Opening in Epitaxial Graphene. Nat. Mater. 2007, 6, $770-775$.

(9) Calizo, I.; Bao, W.; Miao, F.; Lau, C. N.; Balandin, A. A. The Effect of Substrates on the Raman Spectrum of Graphene: Grapheneon-Sapphire and Graphene-on-Glass. Appl. Phys. Lett. 2007, 91, 201904.

(10) Riedl, C.; Coletti, C.; Starke, U. Structural and Electronic Properties of Epitaxial Graphene on $\mathrm{SiC}\left(\begin{array}{llll}0 & 0 & 0 & 1\end{array}\right)$ : A Review of Growth, Characterization, Transfer Doping and Hydrogen Intercalation. J. Phys. D: Appl. Phys. 2010, 43, 374009.

(11) Jain, K.; Klosner, M.; Zemel, M.; Raghunandan, S. Flexible Electronics and Displays: High-Resolution, Roll-to-Roll, Projection Lithography and Photoablation Processing Technologies for HighThroughput Production. Proc. IEEE 2005, 93, 1500-1510.

(12) Kim, B. J.; Jang, H.; Lee, S. K.; Hong, B. H.; Ahn, J. H.; Cho, J. H. High-Performance Flexible Graphene Field Effect Transistors with Ion Gel Gate Dielectrics. Nano Lett. 2010, 10, 3464-3466.

(13) Gomez De Arco, L.; Zhang, Y.; Schlenker, C. W.; Ryu, K.; Thompson, M. E.; Zhou, C. Continuous, Highly Flexible, and Transparent Graphene Films by Chemical Vapor Deposition for Organic Photovoltaics. ACS Nano 2010, 4, 2865-2873. 
(14) Martins, L. G.; Song, Y.; Zeng, T.; Dresselhaus, M. S.; Kong, J.; Araujo, P. T. Direct Transfer of Graphene onto Flexible Substrates. Proc. Natl. Acad. Sci. U.S.A. 2013, 110, 17762-17767.

(15) Verma, V. P.; Das, S.; Lahiri, I.; Choi, W. Large-Area Graphene on Polymer Film for Flexible and Transparent Anode in Field Emission Device. Appl. Phys. Lett. 2010, 96, 203108.

(16) Kim, K. S.; Zhao, Y.; Jang, H.; Lee, S. Y.; Kim, J. M.; Kim, K. S.; Ahn, J.; Kim, P.; Choi, J.; Hong, B. H. Large-Scale Pattern Growth of Graphene Films for Stretchable Transparent Electrodes. Nature 2009, 457, 706.

(17) Wang, Y. Y.; Burke, P. J. A Large-Area and Contamination-Free Graphene Transistor for Liquid-Gated Sensing Applications. Appl. Phys. Lett. 2013, 103, 052103.

(18) Suk, J. W.; Kitt, A.; Magnuson, C. W.; Hao, Y.; Ahmed, S.; An, J.; Swan, A. K.; Golberg, B. B.; Ruoff, R. S. Transfer of CVD-Grown Monolayer Graphene onto Arbitrary Substrates. ACS Nano 2011, 5, 6916.

(19) Ko, P. J.; Takahashi, H.; Koide, S.; Sakai, H.; Thu, T. V.; Okada, H.; Sandhu, A. Simple Method to Transfer Graphene from Metallic Catalytic Substrates to Flexible Surfaces without Chemical Etching. J. Phys.: Conf. Ser. 2013, 433, 012002.

(20) Ghosh, D. S.; Chen, T. L.; Mkhitaryan, V.; Pruneri, V. Ultrathin Transparent Conductive Polyimide Foil Embedding Silver Nanowires. ACS Appl. Mater. Interfaces 2014, 6, 20943-20948.

(21) Hu, L.; Kim, H. S.; Lee, J. Y.; Peumans, P.; Cui, Y. Scalable Coating and Properties of Transparent, Flexible, Silver Nanowire Electrodes. ACS Nano 2010, 4, 2955-2963.

(22) Ghosh, D. S.; Chen, T. L.; Mkhitaryan, V.; Formica, N.; Pruneri, V. Solution Processed Metallic Nanowire Based Transparent Electrode Capped with a Multifunctional Layer. Appl. Phys. Lett. 2013, 102, 221111.

(23) Scardaci, V.; Coull, R.; Lyons, P. E.; Rickard, D.; Coleman, J. N. Spray Deposition of Highly Transparent, Low-Resistance Networks of Silver Nanowires over Large Areas. Small 2011, 7, 2621-2628.

(24) Yang, H.; Jiang, P. Self-cleaning Diffractive Macroporous Films by Doctor Blade Coating. Langmuir 2010, 26, 12598-12604.

(25) Lahiri, J.; Miller, T. S.; Ross, A. J.; Adamska, L.; Oleynik, I. I.; Batzill, M. Graphene Growth and Stability at Nickel Surfaces. New J. Phys. 2011, 13, 025001.

(26) Vanin, M.; Mortensen, J. J.; Kelkkanen, A. K.; Garcia-Lastra, J. M.; Thygesen, K. S.; Jacobsen, K. W. Graphene on Metals: a Van der Waals Density Functional Study. Phys. Rev. B 2010, 81, 081408.

(27) Yoon, T.; Shin, W. C.; Kim, T. Y.; Mun, J. H.; Kim, T. S.; Cho, B. J. Direct Measurement of Adhesion Energy of Monolayer Graphene As-Grown on Copper and Its Application to Renewable Transfer Process. Nano Lett. 2012, 12, 1448-1452.

(28) Shinagawa, T.; Shibata, K.; Shimomura, O.; Chigane, M.; Nomura, R.; Izaki, M. Solution-Processed High-Haze ZnO Pyramidal Textures Directly Grown on a TCO Substrate and the Light-Trapping Effect in $\mathrm{Cu}_{2} \mathrm{O}$ Solar Cells. J. Mater. Chem. C 2014, 2, 2908-2917.

(29) Chiba, Y.; Islam, A.; Komiya, R.; Koide, N.; Han, L. Conversion Efficiency of $10.8 \%$ by a Dye-Sensitized Solar Cell Using a $\mathrm{TiO}_{2}$ Electrode with High Haze. Appl. Phys. Lett. 2006, 88, 223505.

(30) Berginski, M.; Hüpkes, J.; Schulte, M.; Schöpe, G.; Stiebig, H.; Rech, B.; Wuttig, M. The Effect of Front ZnO: Al Surface Texture and Optical Transparency on Efficient Light Trapping in Silicon Thin-Film Solar Cells. J. Appl. Phys. 2007, 101, 074903.

(31) Chen, T. L.; Ghosh, D. S.; Formica, N.; Pruneri, V. Graphene as an Anti-Permeation and Protective Layer for Indium-Free Transparent Electrodes. Nanotechnology 2012, 23, 395603.

(32) Sazonov, A.; Striakhilev, D.; Lee, C. H.; Nathan, A. LowTemperature Materials and Thin Film Transistors for Flexible Electronics. Proc. IEEE 2005, 93, 1420-1428.

(33) Cecchini, M.; Signori, F.; Pingue, P.; Bronco, S.; Ciardelli, F.; Beltram, F. High-Resolution Poly(ethylene terephthalate) (PET) Hot Embossing at Low Temperature: Thermal, Mechanical, and Optical Analysis of Nanopatterned Films. Langmuir 2008, 24, 12581-12586.

(34) Li, S.; Chen, W.; Chu, D.; Roy, S. Self-Aligned High-Resolution Printed Polymer Transistors. Adv. Mater. 2011, 23, 4107-4110. 